\title{
Subsurface-Scattering Calculations via the 3D FDTD Method Employing PML ABC for Layered Media ${ }^{\dagger}$
}

\author{
UĞUR OĞUZ AND LEVENT GÜREL* \\ DEPARTMENT OF Electrical AND ElECTRONICS ENGINEERING \\ BILKENT UNIVERSITY \\ BILKENT, ANKARA, TURKEY \\ (lgureloee.bilkent.edu.tr)
}

\section{Introduction}

The finite-difference time-domain (FDTD) [1-3] method is suitable for solving scattering problems that contain several inhomogeneities [4-7] such as multiple objects of different material properties buried in a layered medium. The advantage of the FDTD method is that the number of unknowns remains the same and a small amount of extra modeling effort is needed for these problems. We have developed a three-dimensional (3D) FDTD computer program that employs pure scattered-field formulation and perfectly matched layers (PML) [8] as the absorbing boundary condition (ABC) of choice. The purposes of this study are to model a subsurface radar and to observe and distinguish between the fields scattered from various buried objects with different parameters such as the size, depth, number, etc.

\section{PML for Layered Media}

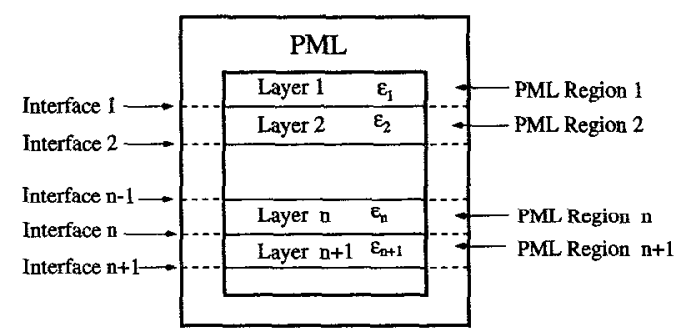

Figure 1: Example of a stratified media and the matching PML ABC.

Consider the dielectric layers indexed as $n$ and $n+1$ separated by the interface indexed as $n$ in Fig. 1. PML layers associated with each dielectric layer can be designed to absorb waves incident from a particular dielectric layer. The permittivity of the layers $n$ and $n+1$

\footnotetext{
'This work was supported in part by NATO's Scientific Affairs Division in the framework of the Science for Stability Programme and in part by the Scientific and Technical Research Council of Turkey (TUBITAK) under contract EEEAG-163.
}

0-7803-4178-3/97/\$10.00 @ 1997 IEEE 
are $\epsilon_{n}$ and $\epsilon_{n+1}$, respectively. The effective permittivity at the interface $n$ is assumed to be $\left(\epsilon_{n}+\epsilon_{n+1}\right) / 2$. The corresponding matching conditions are

$$
\frac{\sigma}{\epsilon_{n}}=\frac{\sigma^{*}}{\mu_{0}}, \quad \frac{\sigma}{\epsilon_{n+1}}=\frac{\sigma^{*}}{\mu_{0}}, \quad \text { and } \quad \frac{2 \sigma}{\epsilon_{n}+\epsilon_{n+1}}=\frac{\sigma^{*}}{\mu_{0}}
$$

for the dielectric layers $n$ and $n+1$, and for the interface $n$, respectively.

\section{Geometry of the Subsurface-Scattering Problem}

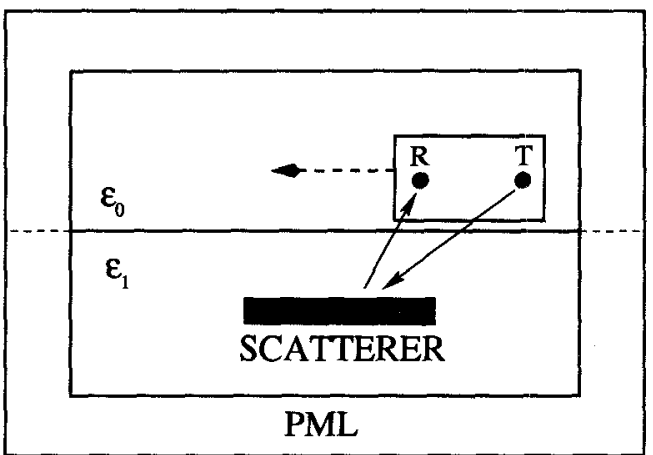

Figure 2: The geometry of a half-space problem with a buried scatterer. A radar unit which contains a transmitter and a receiver travels over the object at a fixed elevation.

A 3D FDTD computational domain containing a vacuum region and a ground region with $\epsilon_{r}=2$ is prepared to simulate the operation of a subsurface radar. The radar is assumed to contain a transmitter and a receiver, which travel together in the radar unit. Other transmitter and receiver configurations [9] can also be modelled. An arbitrary number of perfect electric conductor (PEC) and dielectric objects can be modelled in the ground region. The computational domain is surrounded by PML ABCs to minimize the reflections. The current pulse on the transmitter and the FDTD grid parameters $\Delta x, \Delta y, \Delta z$, and $\Delta t$ are the same as those used by Chew and Weedon [10].

The raw signal received by the receiver is composed of three components: (i) the incident signal, which comes directly from the transmitter, (ii) the reflected signal, which is due to the vacuum-ground interface, and (iii) the scattered signal, which is due to the buried object(s). Although the raw received signal is easy to obtain in practice, it is dominated by the incident and the reflected signals and, therefore, the scattered signal is not easily detectable. For this reason, we subtract the incident and the reflected signals from the raw received signal to obtain the scattered signal. This signal is difficult to obtain in practice, however, we use it as a reference signal in our study. A signal that can be obtained in practice is the "derivative" signal, which can be obtained by differentiating the raw signal with respect to the radar position. The differentiation operation can be approximated using finite differencing, i.e., by subtracting two consecutive raw signals. In the following section, both the reference 
scattering signals and the derivative signals are presented for some sample problems. Both signals are normalized to have a maximum value of unity over all time steps and all radar positions.

\section{Sample Results}
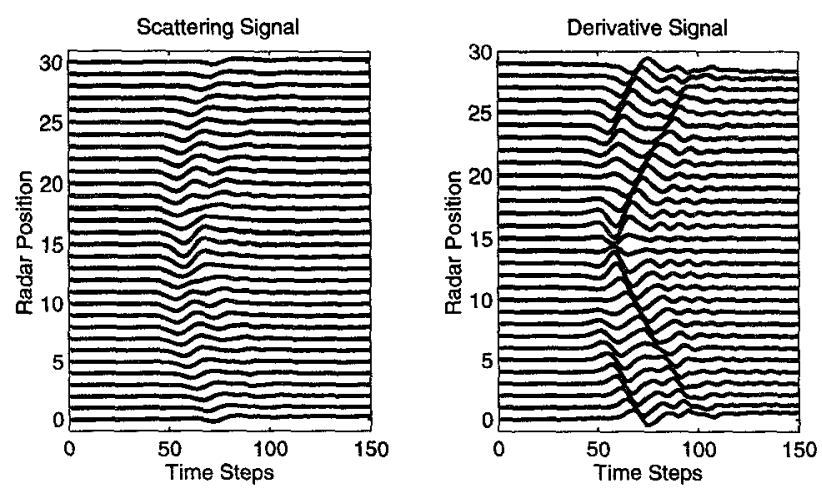

Figure 3: Two single-cell cubes placed horizontally.
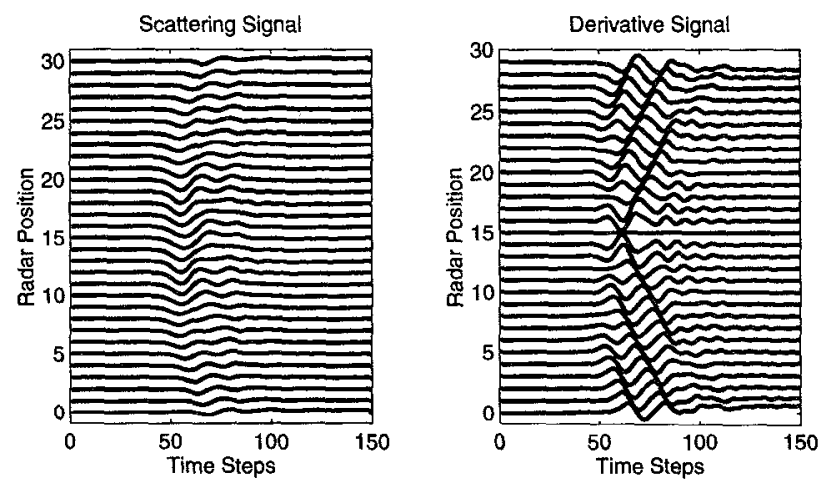

Figure 4: Three single-cell cubes placed horizontally.

Figures 3 and 4 show the FDTD simulation results for two and three, respectively, small single-cell PEC cubes that are horizontally aligned. The PEC scatterers are buried 5 cells under the ground and separated by 8 cells. In Fig. 3, the two objects are identified successfully. On the other hand, it is harder to interpret the scattering-signal results of Fig. 4 to identify the three objects. However, the derivative-signal results are more helpful in this respect. Figure 5 shows the scattering and derivative signals for two small cubes that are vertically aligned 5 and 9 cells under the ground. The scattering effects of these two objects are clearly separated in time due to the difference in their depths. 

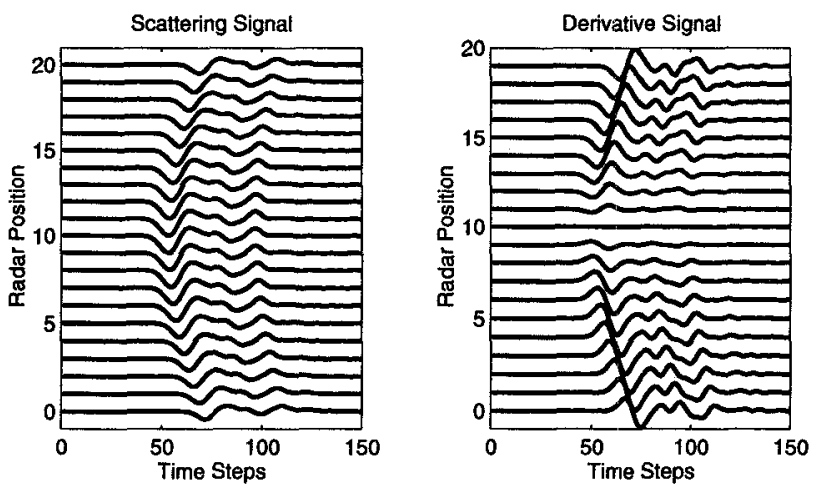

Figure 5: Two single-cell cubes placed vertically.

\section{Conclusions}

3D FDTD method that employs PML ABCs (for layered media) is used to simulate the operation of a subsurface radar. The "derivative" signal, which can easily be obtained in practical systems, is shown to be useful in the identifying the buried objects.

\section{References}

[1] K. S. Yee, "Numerical solution of initial boundary value problems involving Maxwell's equations in isotropic media," IEEE Trans. Antennas Propagat., vol. AP-14, pp. 302-307, Apr. 1966.

[2] A. Taflove, Computational Electrodynamics: The Finite-Difference Time-Domain Method. Boston, MA: Artech House, 1995.

[3] K. S. Kunz and R. J. Luebbers, The Finite Difference Time Domain Method for Electromagnetics. Boca Raton, FL: CRC Press, 1993.

[4] M. Moghaddam. E. J. Yannakakis, W. C. Chew and C. Randall, "Modeling of the subsurface interface radar," J. Electromagnetic Waves and Applications, vol. 5, no. 1, pp. 17-39, 1991.

[5] K. Demarest, R. Plumb, and Z. Huang, "FDTD modeling of scatterers in stratified media," IEEE Trans. Antennas Propagat., vol. AP-43, pp. 1164-1168, Oct. 1995.

[6] K. Demarest, Z. Huang, and R. Plumb, "An FDTD near- to far-zone transformation for scatterers buried in stratified grounds," IEEE Trans. Antennas Propagat., vol. AP-44, pp. 1150-1157, Aug. 1996.

[7] P.B. Wong, G.L. Tyler, J. E.. Baron, E. M. Gurrola, and R. A. Simpson, "A three-wave FDTD approach to surface scattering with applications to remote sensing of geophysical surfaces," IEEE Trans. Antennas Propagat., vol. AP-44, pp. 504-514, Apr. 1996.

[8] J.P. Berenger. "A perfectly matched layer for the absorption of electromagnetic waves, J. Comput. Phys., pp 185-200, Oct. 1994.

[9] D. A. Hill, "Near-field detection of buried dielectric objects," IEEE Trans. Geosci. Remote Sensing, vol. GE-27, pp. 364-368, July 1989.

[10] W. C. Chew and W. H. Weedon, "A 3D perfectly matched medium from modified Maxwell's equations with stretched coordinates," Microwave Opt. Tech. Lett., vol. 7, no. 1.3, pp. 599-604, Sept. 1994. 